\title{
Pulmonary inflammatory myofibroblastic tumour with unusual octreoscan uptake: two reports
}

To the Editors:

Inflammatory myofibroblastic tumour (IMT) is a relatively uncommon lesion and occurs in nearly every site of the body. Patients with IMT are usually asymptomatic, with a solitary nodule or mass detected by routine chest radiograph [1]. Many diagnostic procedures can be applied to make the diagnosis: computed tomography (CT) scan to outline nodule growth and perfusion, $\left[{ }^{18} \mathrm{~F}\right]$-2-fluoro-deoxy-D-glucose (FDG)-PET/CT scan to evaluate metabolic activity and ${ }^{111} \mathrm{In}$-Diethylene triamine pentaacetic acid (DTPA)-D-Phe1 scan (octreoscan) to investigate somatostatin analogue receptors, often present in a variety of neuroendocrine tumours (NETs).

We present two cases of pulmonary IMT clinically misdiagnosed as carcinoid tumours due to a positive octreoscan.

Case 1 was a 52-yr-old female former smoker who was admitted to our hospital with thoracic pain. The electrocardiogram was completely negative for angina or myocardial infarction. Chest radiograph and CT revealed a left hilar nodule, sharply circumscribed, slightly lobulated, with a mild homogeneous increase of density after enhancement. A wholebody FDG-PET/CT scan showed increased tracer uptake of the lung mass (standardised uptake value (SUV) 25) compatible with a malignant lesion. SUVmax was calculated $50 \mathrm{~min}$ after injection and normalised for body weight. After intravenous administration of $111 \mathrm{mBq}$ of $\left[{ }^{111} \mathrm{In}\right]$-octreotide, an abnormal, although low, uptake was present in the left lung hilar region (fig. 1a). Bronchoscopy was negative. On the basis of the CT, FDG-PET/CT and octreoscan, a clinical/radiological diagnosis of a carcinoid tumour, most probably atypical, was postulated. The intraoperative frozen specimen excluded the diagnosis of a carcinoid tumour and the lesion was regarded as a low-grade spindle cell malignancy. A left upper lobectomy with complete lymph node dissection was performed. Tumour size was $3 \mathrm{~cm}$ in the greatest diameter, circumscribed and yellowish on the cut surface. Microscopically the lesion consisted of spindle cells arranged in a storiform pattern and an admixture of inflammatory cells, with a predominance of histiocytes and lymphocytes. The spindle cells had low cellular atypia and no mitotic activity. Immunohistochemical findings are reported in table 1 and somatostatin receptor (SSTR)2A, SSTR3 and SSTR5 staining is shown in figures $1 \mathrm{~b}-\mathrm{d}$. The final diagnosis was IMT. The post-operative course was unremarkable and the patient was discharged from the hospital after 8 days.

Case 2 was a 31-yr-old male former smoker who was admitted to our hospital with a 3-month history of dry cough, low-grade fever, clubbing and bilateral knee, ankle joint stiffness, anaemia and increased erythrocyte sedimentation rate. Past history showed that both a thorough microbiological and immunological analyses were negative. Chest radiograph and CT scan with contrast enhancement revealed a left lower lobe round-shaped solitary lesion with sharp margins, slight
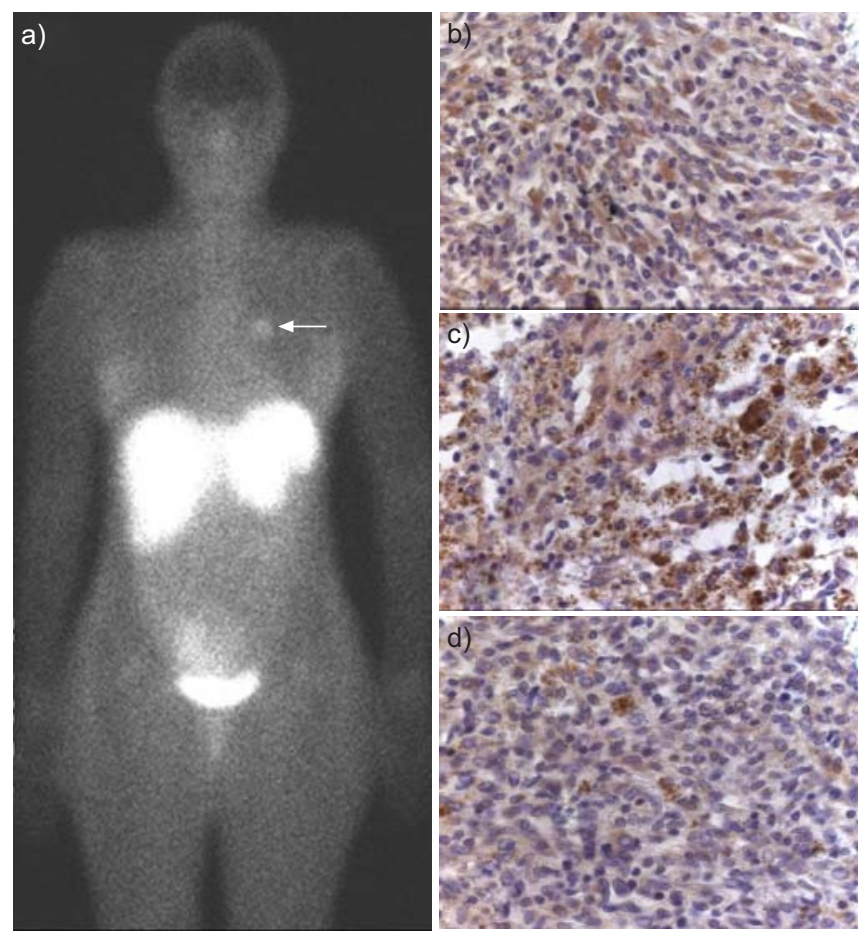

FIGURE 1. (Case 1). Octreoscan uptake in the left hilar region of the lung (a) and immunohistochemistry for SSTRs: scattered neoplastic spindle cells and inflammatory cells strongly marked are well seen (b: SSTR2A, c: SSTR3, d: SSTR5. Original magnification: $\times 200$ ).

inhomogeneous hypodensity and mild, homogeneous enhancement uptake. Bronchoscopy was normal and a wholebody FDG-PET/CT scan revealed a lesion with high hypermetabolism (SUV: 22). SUVmax was measured as described in case 1. Octreoscan showed high tracer uptake of the pulmonary mass. A proposed diagnosis of carcinoids (perhaps atypical) was made.

The patient underwent a left lower lobectomy with complete lymph node dissection. On gross examination, the tumour size was $4 \mathrm{~cm}$ in the greatest diameter, well circumscribed and greyish on the cut surface. At histology, the lesion demonstrated marked cellular proliferation of spindle cells with admixed inflammatory cells mainly represented by plasma cells, lymphocytes and macrophages. Immunohistochemical findings are reported in table 1. The final diagnosis was IMT. The post-operative course showed quick resolution of the systemic symptoms and signs, and the patient was discharged after five days.

IMT is a generic term used for a variety of neoplastic and nonneoplastic entities that have a common histological feature characterised by bland spindle cell proliferation with a prominent, usually chronic, inflammatory infiltrate. 


\begin{tabular}{lcc} 
TABLE 1 & Antibody findings & \\
\hline Antibody & Case 1 & Case2 \\
\hline CD68 & + (macrophages) & + \\
SMA & + (spindle cells) & + \\
ALK1 & - & - \\
Vimentin & + & + \\
Cytokeratin & - & - \\
CD34 & - & - \\
S100 & - & - \\
EMA & - & $-/+$ \\
SSTR2A & + & + \\
SSTR3 & + & $+($ few cells $)$ \\
SSTR5 & + & + (few cells) \\
\hline
\end{tabular}

CD: cluster of differentiation; SMA: smooth muscle actin; ALK1: activin receptor-like kinase 1; EMA: epithelial membrane antigen; SSTR: somatostatin receptor.

The old concept of MATSUBARA et al. [2] which considered most cases of IMT a result of an aberrant repair reaction has now been changed. Since the 1990s, IMT has become known as a distinct entity with peculiar clinical, pathological and molecular characteristics. Under the current classification IMTs are considered to be myofibroblastic; however, smooth muscle actin positivity and other markers of myofibroblastic differentiation cannot always be demonstrated. Macrophagedendritic cell markers have been reported in different series [3] suggesting that IMT may also be considered a proliferative disorder of these cell lines. Thus, as reported by FARRIS et al. [4], the term "inflammatory myfibroblastic tumour" is a misnomer in $\sim 50 \%$ of cases.

Flow cytometry studies have found aneuploid (hyperploid) indices in IMT, leading to the suggestion that it is a neoplasm. Multiple chromosomal abnormalities have been detected in IMT most notably involving the ALK locus on chromosome 2p23. Clonal abnormalities of ALK are usually detected in 50$60 \%$ of anaplastic large cell lymphomas providing further support for the neoplastic nature of IMT. The expression of ALK1 and p80 are reported in $\sim 40 \%$ of IMT cases [1]. Reactivity for p53 has been detected with variable frequency (from $<10 \%$ to $80 \%$ ) and its association with recurrence and malignant transformation has been largely debated [1].

The lungs are frequently involved but the incidence is reported to be $<1 \%$ of all lung tumours. Symptoms are related to tumour localisation; however, many cases are asymptomatic [1]. Of the cases reported in the literature only $19 \%$ were accompanied by some type of systemic symptoms such as fever, anaemia and increased erythrocyte sedimentation rate [5] as were present in one of our reported cases. Previous studies have demonstrated overproduction of large amounts of pro-inflammatory cytokines such as tumour necrosis factor $\alpha$, interleukin (IL)-6 and IL-3; which could be responsible for many systemic symptoms [6]. The prognosis of patients with resected IMT is excellent: $78-100 \%$ of patients were in complete remission after an average follow-up of 3.3 yrs. Intrathoracic recurrence was reported in $5 \%$ of cases and metastasis occurs in $<5 \%$ of cases [1].

The radiological differential diagnosis for IMT occurring as a solitary pulmonary nodule includes primary or secondary neoplasms, haemartoma, chondroma, and granuloma.

In our cases, the patients were studied by conventional chest radiograph, CT, FDG-PET/CT and octreoscan. Few works have described the use of FDG-PET/CT in IMT, reporting different ranges of SUV values (from 5 to $>35$ ) [7, 8]. A larger number of cases could provide strategic information on clinical and morphological/molecular phenotypes. The positive findings of the octreoscan in our cases are related to the immunohistochemical detection of SSTRs on all tumour cell components, either spindle or inflammatory cells. SSTRs are largely expressed in NETs and represent the molecular basis for the clinical use of somatostatin analogues in treatment of NETs and their in vivo localisation. SSTR subtype expression has been detected in other different types of human tumours [9] and in non-neoplastic lesions, particularly immunemediated diseases such as rheumatoid arthritis, Graves' disease, tuberculosis and sarcoidosis [10]. To our knowledge, these are the first documented cases of IMT with positive octreoscan and SSTR immunohistochemistry.

In summary, IMT should be kept in mind when ruling out a diagnosis of a solitary pulmonary nodule, even in cases with positive octreoscan results.

A combination of clinical information, CT, FDG-PET and octreoscan findings could lead to a more precise diagnostic characterisation of pulmonary nodules. Moreover, the confirmation of such findings in larger case series may also give new insights for a targeted therapy.

\section{F. Calabrese*, A. Zuin", E. Brambilla", P. Zucchetta ${ }^{+}$, F. Lunardi*, M. Valente* and F. Rea ${ }^{\#}$}

*Dept of Diagnostic Medical Sciences and Special Therapies, \#Dept of Cardio-Thoracic and Vascular Sciences, and ${ }^{+}$Dept of Nuclear Medicine, University of Padua, Padua, Italy. "Dept of Pathology and Lung Cancer Research Group INSERM U 823, Institut A. Bonniot, Université Joseph Fourier, CHU Michallon, Grenoble, France.

Correspondence: F. Calabrese, Dept of Diagnostic Medical Sciences and Special Therapies, University of Padua Medical School, Via Gabelli 61, 35121 Padua, Italy. E-mail: fiorella. calabrese@unipd.it

Statement of Interest: None declared.

\section{REFERENCES}

1 Yousem SA, Tazelaar HD, Manabe $\mathrm{T}$, et al. Inflammatory myofibroblastic tumour. In: Travis WD, Brambilla E, MullerHermelink HK, Harris CC, eds. Pathology and genetics of tumours of the lung, pleura, thymus and heart. IARC, Lyon, pp. 105-106.

2 Matsubara O, Tan-Liu NS, Kenney RM, et al. Inflammatory pseudotumors of the lung: progression from organizing pneumonia to fibrous histiocytoma or to plasma cell granuloma in 32 cases. Hum Pathol 1988; 19: 807-814. 
3 Pileri SA, Grogan TM, Harris NL, et al. Tumours of histiocytes and accessory dendritic cells: an immunohistochemical approach to classification from the International Lymphoma Study Group based on 61 cases. Histopathology 2002; 41: 1-29.

4 Farris AB 3rd., Mark EJ., Kradin RL, Pulmonary "inflammatory myofibroblastic" tumors: a critical examination of the diagnostic category based on quantitative immunohistochemical analysis. Virchows Arch 2007; 450: 585-590.

5 Chun YS, Wang L, Nascimento AG, et al. Pediatric inflammatory myofibroblastic tumour: anaplastic lymphoma kinase (ALK) expression and prognosis. Pediatr Blood Cancer 2005; 45: 796-801.

6 Fukano R, Matsubara T, Inoue $\mathrm{T}$, et al. Time lag between the increase of IL- 6 with fever and NF- $\kappa$ B activation in the peripheral blood in inflammatory myofibroblastic tumour. Cytokine 2008; 44: 293-297.
7 Sato M, Takasaka I, Okumura T, et al. F-18 fluorodeoxyglucose accumulation in an inflammatory pseudotumor of the spleen. Ann Nucl Med 2007; 21: 521-524.

8 Reddy MP, Menda Y, Floresca J, et al. FDG positron emission tomographic imaging of pseudo-pseudo tumor. Clin Nucl Med 2002; 27: 445-446.

9 Reubi JC, Kappeler A, Waser B, et al. Immunohistochemical localization of somatostatin receptors sst2A in human tumors. Am J Pathol 1998; 153: 233-245.

10 Paran D, Paran H. Somatostatin analogs in rheumatoid arthritis and other inflammatory and immune-mediated conditions. Curr Opin Investig Drugs 2003; 4: 578-582.

DOI: $10.1183 / 09031936.00053609$

\section{Selective apoptosis of lung cancer cells with talc}

\section{To the Editors:}

Lung cancer is the leading cause of cancer deaths worldwide, with adenocarcinoma being the most common cell type. The majority of patients with lung cancer present at an advanced stage of disease with only $15 \%$ being amenable to surgery for cure; treatment is, therefore, palliative. For patients with malignant effusions due to pleural metastasis, chemical pleurodesis is one method by which to prevent recurrence. While there are many intrapleural sclerosing agents, most data pertaining to pleurodesis have been reported with tetracycline derivatives, bleomycin and talc [1].

A number of studies have demonstrated superior efficacy of talc over other sclerosing agents commonly used for the palliation of malignant pleural effusions [2], and talc is the preferred pleurodesis agent according to a survey of chest physicians [3]. Despite talc's wide clinical use, the exact mechanisms for its efficacy as well as its apoptotic effects on lung cancer cells in vitro have not been studied. The objectives of our study were to determine if talc caused apoptosis of lung cancer cells, and to compare talc against other commonly administered intrapleural sclerosing agents by extending the experiments to include bleomycin and doxycyline.

The lung adenocarcinoma cell line (LAC, A549) was purchased from American Type Culture Collection (Rockville, MD, USA). Human pleural mesothelial cells (PMC) characterised by classic cobblestone morphology, cytokeratin and without factor VIII antigen were isolated from pleural fluid obtained via thoracentesis from patients with symptomatic transudative pleural effusions secondary to congestive heart failure, and without evidence of infection. Written informed consent was obtained from all patients and performed in accordance with the protocol approved by the institutional review board (Singapore General Hospital, Singapore).

Both PMC and LAC were maintained in RPMI-1640 culture medium (Gibco Laboratory, Long Island, NY, USA) and 10\% fetal calf serum (Hyclone, Logan, UT, USA) at $37^{\circ} \mathrm{C}$, in $5 \% \mathrm{CO}_{2}$, $95 \%$ air and $100 \%$ humidity.

Talc particles measuring $2 \mu \mathrm{m}$ were prepared using density gradient separation (Ficoll Hypaque ${ }^{\mathrm{TM}}$ Plus; Amersham Pharmacia, Amersham, UK), and confirmed against silicone beads of the same size (Becton Dickinson, San Jose, CA, USA) as reference under an inverted microscope (CK-X31; Olympus, Tokyo, Japan). Talc was applied as a suspension in endotoxinfree normal saline while parenteral formulations of bleomycin and doxycycline were prepared using aseptic techniques. Equal numbers of PMC and LAC were exposed to 25, 50 and $75 \mu \mathrm{g} \cdot \mathrm{mL}^{-1}$ of talc, bleomycin and doxycycline, and cellular apoptosis was measured with propidium iodide staining and flow cytometry at 24, 48 and $72 \mathrm{~h}$. Washed and autoclave sterilised silicone beads at equivalent concentrations were used as parallel control.

At specified time intervals, LAC and PMC were harvested from cultures using $0.125 \%$ trypsin solution, washed with PBS (Gibco) and fixed with ethanol. They were stained with propidium iodide solution $\left(5 \mu \mathrm{g} \cdot \mathrm{mL}^{-1}\right.$; Sigma Aldrich, St Louis, MO, USA) containing $0.1 \%$ triton X-100 and RNase A [4]. DNA contents of target cells were analysed using fluorescence-activated cell sorter Calibur flow cytometer and CellQuest Pro software (Becton Dickinson) as described previously [5], and the sub-G1 population was identified as the marker for apoptosis (fig. 1). Experiments were performed in triplicate at each time-point and for each cell line. Cellular apoptosis measuring $<3 \%$ was considered as baseline rate. Multiple comparisons were performed with ANOVA, and $\mathrm{p} \leqslant 0.05$ was considered statistically significant.

Differences in the apoptosis rates of LAC were observed with graded concentrations of talc, bleomycin, and doxycycline at $24 \mathrm{~h}(\mathrm{p}=0.009), 48 \mathrm{~h}(\mathrm{p}=0.006)$ and $72 \mathrm{~h}(\mathrm{p}=0.01)$. Talc induced apoptosis of LAC in a dose- and time-dependent manner which was superior to bleomycin but inferior to doxycycline (table 1). No apoptosis of PMC was documented with talc but 